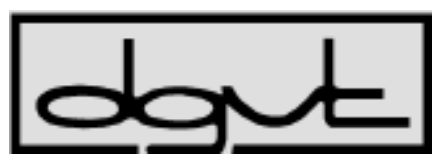

\section{Deutsche Gesellschaft für Verhaltenstherapie e.V. (DGVT)}

Bundesgeschäftsstelle

Postfach 1343

D-72003 Tübingen

Tel. 07071/943494, Fax 943435

E-maildgvt@dgvt.de,www.dgvt.de

\section{Bericht vom 15. Kongress für Klinische Psychologie, Psychotherapie und Beratung vom 5.- 9. März 2004 an der Humboldt-Universität, Berlin}

«Brennpunkt Psychotherapie» - unter diesem Rahmenthema fand im März 2004 der 15. Kongress für Klinische Psychologie, Psychotherapie und Beratung in Berlin statt; erstmals in der Humboldt-Universität im Zentrum der Stadt. Etwa 1200 Personen diskutierten im Rahmen von 34 Symposien über eine breite Vielfalt an Themen rund um die Psychotherapie. Das Spektrum der Themen erstreckte sich von der Frage «Approbation und was dann?» über die Frage nach «Neuen verhaltenstherapeutischen Therapiemethoden in der Behandlung von Suchtproblemen», den multiprofessionellen Blick auf die «Kindheit heute - Anforderungen an die psychotherapeutische Ausbildung, Versorgung und Qualität», die Frage nach den «Handlungsfehlern und Misserfolgen in der Psychotherapie» ... bis hin «Zur Wirkung von Psychotherapiesupervision». Doch nicht nur die Psychotherapie im engeren Sinne stand im Brennpunkt des 15. DGVT- Kongresses; es wäre kein DGVTKongress, wenn unter diesem Rahmenthema nicht über den «kurativen Tellerrand» hinausgeblickt worden wäre: So wurde beispielsweise auch nach Möglichkeiten und Ansätzen der «Prävention und Gesundheitsförderung für Kinder und Jugendliche» gefragt, und das Forum Beratung lud ein zum Gespräch über die «Zukunft von Beratung - Zukunft für Beratung», in dem geographisch wie professionell Grenzen überschritten wurden. Parallel zu den Symposien wurde in einer Vielzahl von Workshops zugehört, diskutiert und geübt.

«Gelehrt und gelernt» wurde auch bereits im Rahmen der Kongresseröffnung und Begrüßung am Freitagabend. Prof. Dr. Grawe eröffnete den Kongress mit einem Vortrag zum
Thema «Von der Verhaltenstherapie zur Neuropsychotherapie». Von den Hausherren der Universität, Prof. Dr. Tenorth und Prof. Dr. Ackermann, erfuhren die KongressteilnehmerInnen einiges über die Geschichte und die - von Finanzmittelknappheit geprägte - gegenwärtige Situation der traditionsreichen Humboldt-Universität. Und - nicht zu vergessen - der Heidelberger HardChor informierte auf außerordentlich unterhaltsame Weise musikalisch über Männerschicksale, Heidegger und den Sinn des Lebens, ... bis hin zur «eigentlichen Zielsetzung von Psychotherapie»: Auch das Fischstäbchen zu befähigen und dazu zu bewegen «weiterzukrabbeln»und, selbstredend, es in diesem Krabbeln zu unterstützen.

Auch der 15. DGVT-Kongress in Berlin machte wieder deutlich, dass das Feld, in dessen Brennpunkt die Psychotherapie im Rahmen des Kongresses stand, ein sehr weites war, welches sich - entsprechend der Tradition der DGVT - mitnichten auf die gesundheitliche oder sogar psychotherapeutische Versorgung begrenzen lässt.

In diesem Sinne wird die inhaltliche Planungsgruppe der DGVT den 16. Kongress für Klinische Psychologie, Psychotherapie und Beratung 2006 in Berlin vorbereiten - und freut sich schon heute darauf, Sie dort begrüßen zu dürfen.

\section{Postkarten-Aktion der DGVT-Landessprecher für eine gemeinsame «Psychotherapeutenkammer Nord»}

Die Postkarten-Aktion der DGVT-Landessprecher Bremens, Hamburgs, Niedersachsens und Schleswig-Holsteins konnte zum 31. März 2004 mit einem beachtlichen Ergebnis abgeschlossen werden: Seit Beginn der Aktion im Januar haben sich insgesamt 1401 Approbierte aus den vier Ländern für einen Zusammenschluss der norddeutschen Landespsychotherapeutenkammern zu einer «gemeinsamen Psychotherapeutenkammer Nord» ausgesprochen. Damit unterstützen knapp über $25 \%$ der insgesamt 5400 Approbierten der vier Landespsychotherapeutenkammern die Forderung nach einer länderübergreifenden Kammerstruktur.

Die Landessprecher der DGVT werden in den nächsten Wochen den Vorständen der jeweiligen Kammern auf den bevorstehenden Kammerversammlungen die zurückgesandten Postkarten überreichen.

Anfang Februar 2004 hatten sich die Vorstände der betroffenen Kammern mit einer gemeinsamen Stellungnahme gegen einen Zusammenschluss der vier norddeutschen Psychotherapeutenkammern ausgesprochen. Diese Stellungnahme sowie die Replik des Vorstands der DGVT und der DGVT-Landes-

\section{KARGER}

Fax +497614520714

E-mail Information@Karger.de

www.karger.com (c) 2004 S. Karger GmbH, Freiburg 
sprecher sind unter $w w w . d g v t . d e$ veröffentlicht. Auch eine abschließende Einschätzung des Ergebnisses sowie aktuelle Informationen über den weiteren Verlauf der Postkarten-Aktion sind dort zu finden.

\section{Ausbildung in Psychologischer Psychotherapie und Kin- der- und Jugendlichenpsychotherapie: Geplante Lehr- gangsstarts 2004}

Lehrgänge in Kinder- und Jugendlichenpsychotherapie beginnen im Herbst 2004 in Bad Schussenried, Berlin, Datteln, Dresden und Hannover/Hildesheim.

Lehrgänge in Psychologischer Psychotherapie beginnen im Sommer 2004 in Berlin und im Herbst 2004 in Dresden, Hamburg und Krefeld. Im Frühjahr 2004 ist ein Lehrgang im Ausbildungszentrum Bonn gestartet; der Einstieg ist noch möglich.

\section{Neue Fortbildungen des Referates für Aus- und Weiterbildung im Bereich Kinder und Jugendliche: Autismustherapie und Lern-Leistungsstörungen}

In Kooperation mit dem Institut für Autismusforschung e.V. (IFA) bietet die DGVT erstmals die berufsbegleitende Seminarreihe «Autismustherapie» an. Die Fortbildung wird in Bremen angeboten; Ragna und Hermann Cordes (IFA) überneh- men die Leitung des Lehrgangs. Die Seminarreihe umfasst neun Veranstaltungen - jeweils Donnerstag bis Samstag - und beginnt im November 2004. Anmeldefrist ist der 20. Oktober 2004. Die Teilnahmegebühren für die gesamte Seminarreihe betragen 1910,- Euro für Mitglieder und 2150,- Euro für Nichtmitglieder. Die Seminarreihe zielt auf (Sozial-)PädagogInnen, HeilpädagogInnen, ErgotherapeutInnen, PsychologInnen, ÄrztInnen, LehrerInnen oder Personen mit vergleichbarer Qualifikation, die mit Menschen mit einer autistischen Störung arbeiten.

Auch die neue Fortbildung «Lerntherapie mit Kindern und Jugendlichen bei Lern-Leistungsstörungen» zielt auf PsychologInnen, Kinder- und JugendlichenpsychotherapeutInnen, SchulpsychologInnen, (Sozial-)PädagogInnen, LehrerInnen und niedergelassene LerntherapeutInnen. Unter der Leitung von Carsten Schmidt wird im Rahmen von drei Veranstaltungen - im Januar, Juni und September 2005 - auf die Diagnoseverfahren im Lern-Leistungsbereich und das therapeutische Vorgehen eingegangen. Veranstaltungsort ist das DGVT-Ausbildungszentrum in Krefeld. Die Teilnahmegebühren betragen pro Veranstaltungstermin 190,- Euro (Mitglieder) bzw. 215,- (Nicht-Mitglieder); der Gesamtpreis für alle drei Veranstaltungen beträgt 540,- Euro (Mitglieder) bzw. 630,- Euro (Nicht-Mitglieder).

Ausführliche Informationen zum Angebot an Aus-, Fort- und Weiterbildung erhalten Sie in der DGVT, Referat Aus- und Weiterbildung, Postfach 13 43, D-72003 Tübingen, Tel. 07071/ 943444, E-mail awk@dgvt.de,www.dgvt.de. 


\section{Workshops beim Internationalen Kongress für Verhaltensmedizin Mainz 25.-28.08.2004}

Der bevorstehende Weltkongress für Verhaltensmedizin in Mainz verspricht ein großer Erfolg zu werden. International besetzte Übersichtsreferate, über 800 Beitragseinreichungen im wissenschaftlichen Programm sowie englischsprachige Workshops durch weltweit anerkannte Experten versprechen spannende Anregungen. Daneben bestehtjedoch auch die Möglichkeit, an deutschsprachigen Workshops, Lunchseminaren, Roundtable-Diskussionen und einer Podiumsdiskussion teilzunehmen, über die wir Sie hiermit besonders informieren möchten.

\author{
NEU ! NEU ! \\ Großes Programm
}

mit internationalen

und deutschsprachigen

Workshops

\section{Deutschsprachige Workshops:}

\section{Mittwoch 25. August -10.00 bis $13.00 \mathrm{Uhr}$}

\begin{tabular}{|c|c|}
\hline Demmel, R. (Universität Münster) & Motivational Interviewing: Wer will schon gute Ratschläge hören? \\
\hline $\begin{array}{l}\text { Herrmanns, N. \& Kubiak, T. (Forschungsinstitut der Diabetes } \\
\text { Akademie Mergentheim) }\end{array}$ & Hypoglykämiewahrnehmung \\
\hline Kropp, P. \& Niederberger, U. (Universität Kiel) & Verhaltensmedizinische Diagnostik und Intervention bei Migräne \\
\hline Dahme, B. \& Ritz, T. (Universität Hamburg) & Verhaltensmedizin des Asthma \\
\hline $\begin{array}{l}\text { Beisel, S. \& Böse, R. (Medizinisch-Psychosomatische Klinik } \\
\text { Roseneck, Prien) }\end{array}$ & $\begin{array}{l}\text { Verhaltensmedizinische Interventionen bei Anorexia und Bulimia } \\
\text { nervosa }\end{array}$ \\
\hline $\begin{array}{l}\text { Knickenberg, R \& Wiehn, T. (Psychosomatische Klinik Bad } \\
\text { Neustadt \& Salus Klinik, Friedrichsdorf) }\end{array}$ & VT bei Schmerzpatienten \\
\hline \multicolumn{2}{|c|}{ Mittwoch 25. August - 14.00 bis 17.00 Uhr } \\
\hline Klinkenberg, N. (Parkklinik Bad Bergzabern) & Verhaltenstherapie und körperorientierte Verfahren \\
\hline Hedlund, S. (Med.-Psychosom. Klinik Roseneck, Prien) & $\begin{array}{l}\text { Kognitive und verhaltenstherapeutische Interventionen bei } \\
\text { Zwangserkrankungen }\end{array}$ \\
\hline Korn, H.-J. (Med.-Psychosom. Klinik Roseneck, Prien) & Temporomandibuläre Dysfunktionen und Biofeedback \\
\hline Timmer, B. (Med.-Psychosom. Klinik Roseneck, Prien) & Biofeedback bei somatoformen Störungen \\
\hline $\begin{array}{l}\text { Vogel, H., Krauth, C. \& Wasem, J. (Universität Würzburg, } \\
\text { Medizinische Hochschule Hannover und Universität Essen) }\end{array}$ & Kosten und Nutzen von verhaltensmedizinischen Interventionen \\
\hline Kraemer, S. (Technische Universität München) & $\begin{array}{l}\text { Kognitive Verhaltenstherapie für persistierende Symptome der } \\
\text { Schizophrenie }\end{array}$ \\
\hline Gaab, J. (Universität Zürich) & Stressbewältigungstraining in der Prävention und Intervention \\
\hline \multicolumn{2}{|c|}{ Samstag 28. August - 14.00 bis 17.00 Uhr } \\
\hline Legenbauer, T. \& Vocks, S. (Universitäten Mainz \& Bochum) & Body-Image Therapie bei Anorexia und Bulimia nervosa \\
\hline Hohagen, F. (Universität Lübeck) & $\begin{array}{l}\text { Verhaltenstherapie und Psychopharmakotherapie bei } \\
\text { Zwangsstörungen }\end{array}$ \\
\hline Fehm-Wolfsdorf, G. (Lübeck) & Mit Hausaufgaben in der Verhaltensmedizin arbeiten \\
\hline Heinrichs, M. (Universität Zürich) & $\begin{array}{l}\text { Psychobiologie, Diagnostik und Intervention bei akuter } \\
\text { Belastungsreaktion }\end{array}$ \\
\hline $\begin{array}{l}\text { Schedlowski, M. \& Göbel, M. (Eidgenössisch-Technische } \\
\text { Hochschule Zürich) }\end{array}$ & Psychoneuroimmunologie in der Verhaltenstherapie \\
\hline
\end{tabular}




\section{Mittwoch 25.August - 17.15 bis 18.15 Uhr}

ZUM VERHÄLTNIS ZWISCHEN VERHALTENSMEDIZIN UND PSYCHOSOMATIK

Diskutanten: Rudolf, G. (Universität Heidelberg) \& Birbaumer, N. (Universität Tübingen); Moderation: Ehlert, U. (Universität Zürich)

Roundtable (deutsch)

Samstag 28. August

14.30 bis $15.30 \mathrm{Uhr}$

NEUROBIOLOGY AND PSYCHOTHERAPY: Prof. Dr. Bauer (Universität Freiburg), Prof. Dr. Braun (Universität Magdeburg), Prof. Dr. Ehlert (Universität Zürich), Prof. Dr. Sachsse (Universität Göttingen)

Lunch Seminars (deutsch)

\begin{tabular}{|l|l|l|}
\hline $\begin{array}{l}\text { Donnerstag 26.August } \\
\text { 13.15. bis 14.30 Uhr }\end{array}$ & $\begin{array}{l}\text { Organisation: } \\
\text { Wiehn, T. \& Rüddel, H. }\end{array}$ & Verhaltensmedizin bei körperlichen Erkrankungen \\
\hline $\begin{array}{l}\text { Freitag 27. August } \\
\text { 13.15 bis 14.30 Uhr }\end{array}$ & $\begin{array}{l}\text { Organisation: } \\
\text { Rüddel, H. \& Wahl, R. }\end{array}$ & $\begin{array}{l}\text { Konzepte der Verhaltensmedizin in Deutschland über die } \\
\text { letzten 20 Jahre, aktueller Stand und Ausblick für die Zukunft }\end{array}$ \\
\hline $\begin{array}{l}\text { Samstag 28. August } \\
\text { 13.15 bis 14.30 Uhr }\end{array}$ & $\begin{array}{l}\text { Organisation: } \\
\text { Rief, W. }\end{array}$ & Somatoforme Störungen \\
\hline $\begin{array}{l}\text { Samstag 28.August } \\
\text { 13.15 bis 14.30 Uhr }\end{array}$ & $\begin{array}{l}\text { Organisation: } \\
\text { Limbacher, A \& Rüddel, H. }\end{array}$ & Essentials of Psychosomatic Rehabilitation \\
\hline
\end{tabular}

Patientenseminar Reizdarm (deutsch)

Samstag 28. August 10.00 bis $14.00 \mathrm{Uhr}$

Leitung: Cuntz, U. (Klinik Roseneck, Prien)

Workshops (English)

\begin{tabular}{|c|c|}
\hline \multicolumn{2}{|r|}{ Wednesday 25 Aug - 10.00-13.00 } \\
\hline Kaptein, Ad (NL) & Illness cognitions in chronic somatic disorders \\
\hline Evers, Kerry E. (USA) & $\begin{array}{l}\text { Developing interventions based on the Transtheoretical Model Of Change: } \\
\text { Multi-national lessons learned }\end{array}$ \\
\hline Chalder, Trudie (UK) & A cognitive-behavioural model for understanding \& treating subjective health complaints \\
\hline Llabre, Maria M. (USA) & Applications of structural equation models in behavioral medicine research \\
\hline Túry, Ferenc (Hungary) & $\begin{array}{l}\text { Communicative aspects of home visits - environmental psychology of psychosomatic } \\
\text { disorders }\end{array}$ \\
\hline Sabate, E. \& Hotz, S. (Switzerland) & Management of adherence to anti-retrovirals professionals' adherence-enhancing skills \\
\hline \multicolumn{2}{|r|}{ Wednesday 25 Aug - 14.00 - 17.00} \\
\hline $\begin{array}{l}\text { Lindahl, Bernt \& Absetz, Pilvikki } \\
\text { (Sweden, Finland) }\end{array}$ & Stress, metabolic syndrome and health behaviour change \\
\hline Lindenmeyer, Johannes (Germany) & Making sense: Motivating patients with alcohol problems \\
\hline Chalder, Trudie (UK) & $\begin{array}{l}\text { Family focused cognitive behavioural treatment of chronic fatigue syndrome and distress in } \\
\text { adolescents }\end{array}$ \\
\hline Van Lenthe, Frank \& Twisk, Jos (NL) & Multilevel modelling in health-related behavioural research \\
\hline $\begin{array}{l}\text { Pekka, Oja \& Sjöström, Michael } \\
\text { (Finland, Sweden) }\end{array}$ & $\begin{array}{l}\text { Using the International Physical Activity Questionnaire (IPAQ): Collection, analyses and } \\
\text { presentation of population data on physical activity }\end{array}$ \\
\hline Moss-Morris, Rona (New Zealand) & Cognitive behavioural management of irritable bowel syndrome in primary care \\
\hline $\begin{array}{l}\text { Smith, Roselyn G. \& Antoni, } \\
\text { Michael H. (USA) }\end{array}$ & $\begin{array}{l}\text { Stress management and relaxation training for women with breast cancer: Scientific } \\
\text { foundations and implementation }\end{array}$ \\
\hline $\begin{array}{l}\text { Keltikangas-Järvinen, Liisa } \\
\text { (Finland) }\end{array}$ & $\begin{array}{l}\text { Relation between innate temperament, stress vulnerability and CHD risk } \\
\text { Dieser Workshop findet am Samstag, 28. August, } 17.00-19.00 \text { Uhr statt. }\end{array}$ \\
\hline
\end{tabular}

Bei Besitz einer Kongresskarte oder Tageskarte ist die Teilnahme an den deutschen Workshops kostenfrei, bei internationalen Workshops fällt eine Zusatzgebühr an 